\title{
Meningioma intraventricular. Presentación de un caso en la infancia
}

\author{
C.L. Marrero; J. Domínguez*; R. Ramos; J. Gómez y J.A. Lourido*
}

Departamento de Pediatría y Servicio de Neurocirugía*.Hospital Universitario Nuestra Señora de Candelaria. Santa Cruz de Tenerife.

\section{Resumen}

Los meningiomas son tumores poco frecuentes en la infancia, pero, de presentarse en este grupo de edad, la localización intraventricular es más prevalente que en adultos.

Presentamos el caso de una paciente de 5 años que debutó clínicamente con síndrome de hipertensión intracraneal con parálisis del VI par izquierdo. En la tomografía computarizada y en las imágenes de resonancia magnética se detectó una masa bien delimitada localizada en el interior del ventrículo lateral derecho. En ambas pruebas diagnósticas la tumoración captaba contraste de forma intensa y homogénea. La lesión se abordó quirúrgicamente a través de la circunvolución temporal media y fue extirpada totalmente. El estudio anatomopatológico tipificó el tumor como meningioma atípico.

PALABRAS CLAVE: Meningioma. Ventrículo lateral. Infancia.

Intraventricular meningioma: case report in infancy

\section{Summary}

Meningiomas are infrequent tumours in infancy but when they appear in this age group, the intraventricular location is more frequent than in adults.

We report the case of a 5-year-old girl with intracranial hypertension and a VI nerve palsy. Computed tomography and magnetic resonance imaging of the brain revealed a tumoral lesion involving the right lateral ventricle with intense enhancement after contrast administration. It was completely resected through a mean temporal circunvolution route. Histological diagnosis was of atypical meningioma.

KEY WORDS: Meningioma. Lateral ventricle. Infancy.

Recibido: 14-10-04. Acepatdo: 03-01-05

\section{Introducción}

Los tumores del SNC son la primera localización de tumores sólidos en la infancia. Se describen dos picos de incidencia de dichos tumores. El primero ocurre en torno a la primera década de la vida y supone 2,2 a 2,5 casos por 100.000 niños por año, con una muy discreta preferencia por el sexo masculino $(1,1: 1)$. El segundo es mucho más amplio abarcando desde la tercera a la cuarta década, con su punto álgido a partir de los 60 años $^{36}$. Dentro del primer pico predominan la neoplasias del SNC de estirpe embrionaria con una relativa ausencia de gliomas. Esto persiste hasta la adolescencia temprana, incrementándose a partir de aquí la incidencia de tumores del SNC típicamente de adultos. Estos diferentes patrones, en cuanto a incidencia e histología, sugieren que los tumores del SNC en niños podrían tener diferencias etiológicas en comparación con los que aparecen en adultos ${ }^{33}$.

Los meningiomas constituyen una rareza en la infan$\mathrm{cia}^{25}$. Se diagnostican con mayor frecuencia en mujeres en torno a la quinta década de la vida, y constituyen entre el 13 y el $27 \%$ de los tumores intracraneales primarios. En niños representan del 1 al $4 \%$ de los tumores intracraneales ${ }^{3,42}$.

Shaw describe por primera vez en 1854 los meningiomas intraventriculares, que constituyen del 0,5 al $15 \%$ de los meningiomas intracraneales en distintas series en adultos ${ }^{6,19}$. Su incidencia en la infancia es proporcionalmente superior, siendo el $15 \%$ intraventriculares, asentando preferentemente a nivel de los ventrículos laterales $^{2,4,13,14,17,18,24,27,29,40}$.

Presentamos el caso de una niña de cinco años con un meningioma del ventrículo lateral y se revisa la literatura al respecto.

\section{Caso clínico}

Paciente de 5 años y 7 meses de edad, sin antecedentes personales o familiares de interés, ingresó en nuestro

Abreviaturas. RM: resonancia magnética. SNC: sistema nervioso central. TAC: tomografía computarizada. 


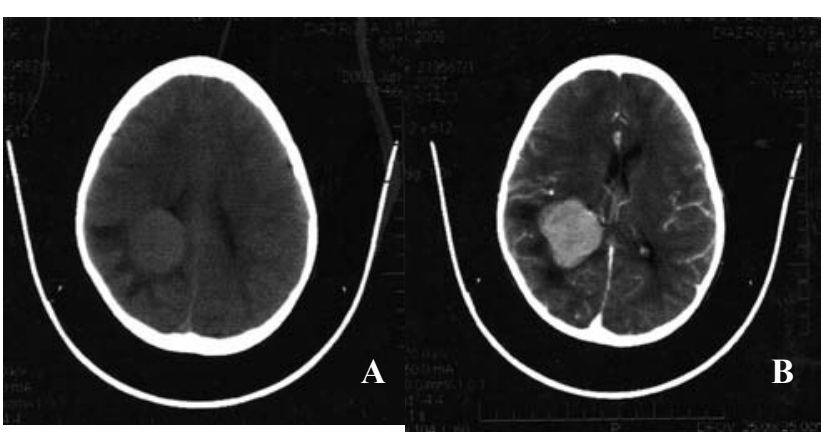

Figural (A y B). Imágenes de TAC sin y con contraste que muestran una lesión hiperdensa intraventricular que capta contraste intensamente.

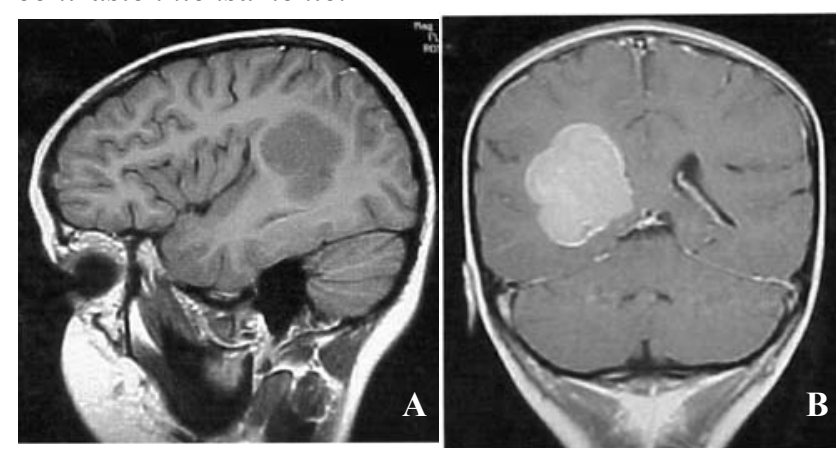

Figura 2 (A y B). Imágenes de resonancia magnética sin y con contraste que muestran una lesión isointensa en T1 con realce homogéneo localizada a nivel del atrio ventricular derecho.

hospital el 12 de junio de 2002 por presentar un cuadro de 10 días de evolución consistente en cefalea de predominio vespertino, al que se añade posteriormente estrabismo convergente del ojo izquierdo y diplopia. En la exploración neurológica nos encontramos con una niña despierta, colaboradora y conectada ambientalmente, que presentaba estrabismo convergente de ojo izquierdo con motilidad ocular conservada y nistagmo horizontal a la mirada forzada hacia la derecha.

La analítica de sangre, bioquímica y radiografía de tórax resultaron normales. La TAC y RM de cráneo (Figs.1 y 2) mostraron una masa bien delimitada localizada en el interior del ventrículo lateral derecho a nivel del atrio y asta temporal, de densidad homogénea y de 6 por 2,5 cms, con importante edema perilesional y que mostraba intensa captación tras la administración de contraste intravenoso.

La paciente fue intervenida mediante abordaje transtemporal a través de la circunvolución temporal media con extirpación macroscópicamente completa de la tumoración.

El estudio histopatológico evidenció una proliferación neoplásica meningotelial con patrón predominantemente meningoteliomatoso y elevada celularidad, tipificándose el tumor como meningioma atípico (Fig. 3). Llamaba la aten-

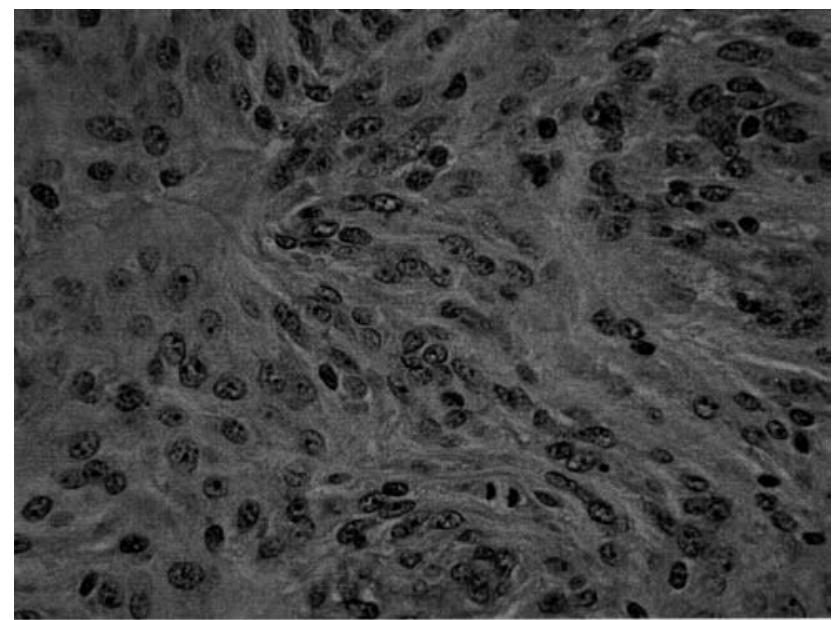

Figura 3. Células tumorales con núcleos grandes y nucleolos prominentes. En la porción inferior se observa una mitosis. (HE $x$ 400).

ción la presencia de una elevada actividad mitótica que, en alguna zona, llegaba a ser de 2-3-mitosis en 10 campos de gran aumento, presentando las células proliferantes núcleos más voluminosos y nucleolo prominente. La actividad del anticuerpo Ki-67, medida de forma aleatoria en la totalidad del tumor, oscilaba en torno al 18-20\%.

La evolución clínica de la niña fue favorable, siendo dada de alta hospitalaria a los diez días de la intervención quirúrgica con un mínimo estrabismo convergente de ojo derecho. A pesar de no haber sido sometida a tratamientos complementarios, en la actualidad, la paciente se encuentra asintomática y no se han evidenciado signos de recidiva en los estudios de imagen de control.

\section{Discusión}

Los meningiomas son tumores inusuales en la infancia. En la serie de Cushing y Eisenhart ${ }^{3}$, de los 313 meningiomas registrados, únicamente $6(1,9 \%)$ debutaron en edades inferiores a los 21 años. Erdinçler et al ${ }^{11}$ encontraron 29 casos $(2,4 \%)$ de 1200 tumores intervenidos en la población pediátrica. En relación a su incidencia según sexos, algunos autores describen un cierto predominio en el sexo masculino ${ }^{10,35,36}$, así como localizaciones atípicas en comparación con adultos. En paciente afectos de neurofibromatosis su incidencia, al igual que la de otros tumores del sistema nervioso central, es mayor ${ }^{11,13}$. Son tumores de lento crecimiento, siendo el tiempo desde el inicio de los síntomas hasta el diagnóstico definitivo de incluso años ${ }^{7}$, y sus manifestaciones clínicas pueden ser intermitentes.

La clínica de estos tumores suele ser secundaria al aumento de presión intracraneal, teniendo en cuenta que en los lactantes, donde aún no se han cerrado los suturas craneales, el aumento del perímetro cefálico puede ser el 
primer signo guía. Los déficits neurológicos focales y las crisis convulsivas también son frecuentemente reportados en la literatura ${ }^{13}$. Las cefaleas suelen ser intermitentes, difusas y homolaterales, desencadenadas ante distintos movimientos, que el paciente acaba evitando. Una vez instaurada la hipertensión intracraneal, la cefalea se vuelve continua y los vómitos frecuentes ${ }^{7}$. Los síntomas oculares se manifiestan subjetivamente como una disminución de la agudeza visual en un $50 \%$ de los casos, detectándose papiledema en 2 de cada 3 casos en la exploración del fondo de ojo. Los trastornos del lenguaje aparecen, aproximadamente, en el $40 \%$ de los tumores que afectan al hemisferio dominante. También pueden aparecer alteraciones psiquiátricas inespecíficas tales como depresión, desorientación y transtornos del comportamiento.

Las hemorragias intraventriculares constituyen una rareza, pero se han descrito como forma de debut en alguna ocasión'. El sangrado puede tener lugar al espacio subaracnoideo, subdural o intraparenquimatoso, aunque en la mayoría de los casos tiene lugar hacia el interior del propio tumor originando un ensanchamiento del mismo y una exacerbación brusca de la sintomatología $a^{1,30,37}$. Algunos autores consideran que este fenómeno se produce únicamente en aquellos tumores altamente vascularizados en los que existen grandes canales vasculares limitados por paredes endoteliales adelgazadas ${ }^{30}$.

En nuestro caso, la instauración de la clínica fue aguda ya que la paciente no refería sintomatología previa a la afectación del VI par craneal izquierdo. Ante los hallazgos clínicos, son las pruebas radiológicas las que delimitan los posibles diagnósticos diferenciales. La TAC y RM mostraron una masa bien delimitada localizada en el interior del ventrículo lateral derecho que captaba contraste de forma homogénea sin detectarse calcificaciones en su interior. Tanto un tumor de plexos coroideos como un ependimoma que envolviese el trígono del ventrículo lateral derecho como un meningioma intraventricular podrían ser compatibles con esa imagen. Los plexopapilomas suelen asociarse a hidrocefalia y con mayor frecuencia presentan calcificaciones en la TAC. Los meningiomas se muestran como una lesión hiperdensa bien definida, con edema variable, y se calcifican en menor medida. En las imágenes de resonancia magnética, los plexopapilomas aparecen hipointensos en $\mathrm{T} 1$ e hiperintensos en T2 mientras que los meningiomas suelen ser isointensos con el resto del parénquima cerebral. Ambos captan contraste intensamente y de manera homogénea. Los ependimomas suelen presentar densidad o intensidad de señal mixta y el realce tras la administración de contraste no suele ser homogénea a diferencia de meningiomas y plexopapilomas.

El tratamiento de elección de los meningiomas intraventriculares es la resección tumoral completa, existiendo distintas vías de abordaje $e^{28}$. Entre ellas destaca la transcallosa ${ }^{20}$, la parietooccipital posterior ${ }^{26}$ y la transtemporal a través de la circunvolución temporal media ${ }^{8}$. Aquellos tumores localizados en el asta frontal y próximos al foramen de Monro pueden abordarse por vía transfrontal, siendo la vía trascallosa más usada en tumores no muy voluminosos, situados a nivel del hemisferio dominante. El abordaje elegido en nuestro caso fue el transcortical a través de la circunvolución temporal media. Independientemente de la vía elegida, el tumor se fragmenta en trozos a partir de la porción intracapsular, siendo rigurosos con la hemostasia.

Los déficits neurológicos secundarios a la intervención varían en función de la localización tumoral y del abordaje quirúrgico. Así el daño visual, o los trastornos disfásicos, aparecerían en tumores que requieran abordaje parietooccipital o en los localizados en el hemisferio dominante respectivamente ${ }^{9}$. La mortalidad perioperatoria referida en la literatura varía considerablemente desde el 5,2 que registró Guidetti et al ${ }^{15}$ hasta el $50 \%$ de Tarlov ${ }^{39}$. En las series revisadas en la infancia este amplio rango de mortalidad se mantiene ${ }^{5,12}$.

El diagnóstico anatomopatológico en nuestro caso fue de meningioma atípico. Este tipo de meningioma presenta hipercelularidad, crecimiento anárquico o en sábana, necrosis local o geográfica $y$, entre los hallazgos citológicos, destacan un índice citoplasma-núcleo elevado, cromatina densa y nucleolos prominentes. Las imágenes de atipia pueden ser focales o extensas. El índice de actividad mitótica es también significativo, aunque puede variar de una región a otra. Las mitosis ocasionales pueden aparecer en cualquier tipo de meningioma, pero cuando aparecen en mayor número debemos tenerlo en cuenta, a partir de 10 mitosis en 10 campos de gran aumento. La invasión cerebral es, por definición, la principal característica de malignidad, más que la atipia citológica en sí, y aunque estos meningiomas, a menudo, tienen un crecimiento adyacente, cualquier infiltración lo suficientemente profunda o extensa podría suponer un importante factor de invasión tisular, tal y como refiere Perry et al. ${ }^{31}$ en su serie de 116 pacientes diagnosticados de "meningioma maligno". Los índices de ploidía y los marcadores de proliferación celular son útiles para diferenciar un meningioma atípico de uno benigno. Así, el índice Ki-67 está elevado en el meningioma maligno, aunque no se trata de un dato específico puesto que los valores se solapan en los casos de meningioma no anaplásico recurrente, anaplásico y común no recurrente ${ }^{32,34}$. También la presencia de índices de tinción con bromodesoxiuridina mayores de un $5 \%$ se han asociado a un comportamiento tumoral más agresivo ${ }^{22}$.

Es difícil especificar la probabilidad de recurrencia de un meningioma atípico, debido en parte a la definición variable de meningioma atípico en distintas series publicadas, en las cuales se incluyen incluso hemangioperici- 
tomas. Según Haltia y Servo ${ }^{16}$, la recurrencia a los cinco años tras haber realizado una resección total del tumor fue del 3\% para los meningiomas clásicos bien diferenciados, $38 \%$ para las lesiones atípicas y $78 \%$ para los tumores anaplásicos ${ }^{16}$. En las series publicadas por Mahmood y $\mathrm{Caccamo}^{23}$, tras la resección total del tumor la recurrencia fue del 50, 67 y $67 \%$ respectivamente.

En los casos en los que no pueda realizarse una resección tumoral completa algunos autores defienden el uso de la radioterapia adyuvante, dependiendo sobre todo del patrón histológico tumoral ${ }^{4,38}$. De hecho, algunas series publicadas ponen de manifiesto una supervivencia 8 veces mayor en pacientes con meningioma maligno sometidos a radioterapia post-operatoria en comparación con aquellos que únicamente fueron intervenidos quirúrgicamente ${ }^{21}$. Está por determinar aún su papel en el tratamiento de los meningiomas, sobre todo en los recurrentes, donde una de las limitaciones para su aplicación es la vulnerabilidad que ya presenta el tejido cerebral previamente sometido a daño quirúrgico ${ }^{41}$.

\section{Bibliografía}

1. Askenazy, H.M., Behmoaram, A.D.: Subarachnoid hemorrahage in meningiomas of the lateral ventricle. Neurology 1960; 10: 484-489.

2. Baumgartner, J.E., Sorenson, J.M.: Meningiomas in the pediatric population. J. Neurooncol 1996; 29: 223-228.

3. Cushing, H., Eisenhardt, L.: Meningiomas: Their classification, regional behaviour, life history, and surgical end results. Springfield, III: Charles C Thomas 1938; pp: 133154.

4. Chan, R.C., Thompson, G.B.: Intracranial meningiomas in childhood. Surg Neurol 1984; 21: 319-322.

5. Davidson, G.S., Hope, J.K.: Meningeal tumors in childhood. Cancer 1989; 63: 1205-1210.

6. Davini, V., Baratta, F.: Meningiomi dei ventricoli laterale. Minerva Neurochir 1963; 18: 520-529.

7. Delandsheer, J. M.: Tumeurs des ventricules latéraux. Systeme Nerveux, 1968; 3: 1-6.

8. De la Torre, E., Alexander, E., Davids, C.H., et al.: Tumors of the lateral ventricles of the brain. Report of 8 cases with suggestion for clinical management. J. Neurosurg 1963; 20: 461-470.

9. Domínguez, J., Lobato R.D., Rivas, J.J., et al.: Meningiomas del ventrículo lateral. Presentación de siete casos. Neurocirugía 1990; 1: 359-365.

10. Drake, J.M., Hoffman, H.J.: Meningiomas in children. En Al-Mefty O (ed). Meningiomas. New York: Raven Press 1991; pp: 145-152.

11. Erdinçler, P., Lena, G., Sarioglu, A.C., et al.: Intracranial meningiomas in children: review of 29 cases. Surg Neurol 1998; 49: 136-140.
12. Ferrante, L., Acqui, M., Artico, M., et al.: Cerebral meningiomas in children. Childs Nerv Syst 1989; 5: 83-86.

13. Germano, I.M., Edwards, M.S.B., Davis, R.L., et al.: Intracranial meningiomas of the first two decades of life. J. Neurosug 1994; 80: 447-453.

14. Gori, G., Nucci, V.: Meningiomi dell'infanzia e dell'eta evolutiva. Considerazioni statische e cliniche. Minerva Neurochir 1963; 7: 119-124.

15. Guidetti, B., Delfini, R., Gagliardi, F., et al.: Meningiomas of the lateral ventricles. Clinical, neuroradiologic and surgical considerations in 19 cases. Surg. Neurol 1985; 24: 364-370.

16. Haltia, M., Servo, A.: Atypical and anaplastic meningiomas: radiology, surgery, radiotherapy and outcome. Surg Neurol 1986; 25: 233-242.

17. Herz, D.A., Shapiro, K., Shulman, K.: Intracranial meningiomas of infancy, childhood and adolescence. Child's Brain 1990; 7: 43-56.

18. Huang, P.P., Doyle, W.K., Abbott, I.R.: Atypical meningioma in a third ventricle in a six year old boy. Neurosurgery $1993 ; 33: 312-315$.

19. Huang, Y.S., Araki, C.: Angiographic confirmation of lateral ventricles meningiomas. A report of 5 cases. J. Neurosurg 1954; 11: 337-352.

20. Jung, C.L., Nutik, S.L.: Surgical approaches to intraventricular meningiomas of the trigone. Neurosurgery 1985; 3: 416-420.

21. King, D.L., Chan, C.H., Pool, J.L.: Radiotherapy in the management of meningiomas. Acta Radiol. Ther Phys Biol 1996; 5: 26-33.

22. Lee, K.S., Hoshino, T., Rodríguez, L.A., et al.: Bromodeoxyuridine labeling study of intracranial meningiomas proliferative potencial and recurrence. Acta neuropathol (Berl) 1990; 80: 311-317.

23. Mahmood, A., Caccamo, D.V., Tomecek, F.J., et al.: Atypical and malignant meningiomas: A Clinicopathological review. Neurosurgery 1993; 33: 955-963.

24. Markwalder, T.M., Seiler, R.W., Markwalder, R.V., et al.: Meningioma of the anterior part of third ventricle in a child. Surg Neurol 1979; 12: 29-32.

25. Martinez-Lage, J.F., Poza, M., Alcaraz, J.,et al.: Giant meningioma of the III ventricle in a child: case report and review of the literature. Childs Nerv Syst 1993; 9: 306-308.

26. McCarthy, C.S., Piepgrass, D.G., Ebersold, M.J.: Meningeal tumors of the brain in young men. Neurological Surgery. Philadelphia. Saunders. 1982; p: 2955.

27. Merten, D.F., Gooding, C.A., Newton, T.H., et al.: Meningiomas in childhood and adolescence. Journal Paediatrics 1974; 84: 696-670.

28. Mircevski, M., Mircevska, D., Bojadziev, I., et al.: Surgical treatment of intraventricular meningioma in childhood. Acta Neurochir 1985; 35 (suppl): 89-91.

29. Molleston, M.C., Moran, C.J., Roth, A.: Meningioma. 
Pediatr Neurosurg 1994; 21: 195-200.

30. Modesti, L.M., Binet, E., Collins,G.: Meningiomas causing spontaneus intracranial hematomas. J. Neurosurg 1976; 45: 437-442.

31. Perry, A., Scheithauer, B.W., Stafford, S.L., et al.: "Malignancy" in meningiomas. A clinicopathologic study of 116 patiens with grading implications. Cancer 1999; 85: 20462065.

32. Perry, A., Stafford, S.L., Scheithauer, B.W., et al.: Meningioma grading. An analysis of histologic parameters. Am J Surg Pathol 1997; 21: 1455-1465.

33. Pizzo, P., Poplack. D.: Principles and Practice of Pediatric Oncology. Philadelphia; JB Lippincott Company, 1993; pp.633-681.

34. Roggendorf, W., Schuster, T., Peiffer, J.: Proliferative potencial of meningiomas determine with the monoclonal antibody Ki-67. Acta Neuropathol (Berl) 1987; 73: 361-364.

35. Sano, K., Wakai, S., Ochiai, C., et al.: Characteristics of intracranial meningiomas in childhood. Child's Brain 1981; 8: $98-106$.

36. Schoenberg, B., Schoenberg D., Christine, B., et al.: The epidemiology of primary intracranial neoplasms of childhood: A population study. Mayo Clin Proc 1976; 51:51.
2005; 16: 523-527

37. Skultery, F.M.: Meningioma simulating ruptured aneurysm. Case report. J. Neurosurg 1968; 28: 380-382.

38. Spallone, A., Gagliardi, F., Vagnozzi, R.: Intracranial meningiomas related to external cranial radiation. Surg Neurol 1979; 12: 153 .

39. Tarlov, E.: Meningiomas of the lateral ventricles. Contemp. Neurosurg 1987; 24: 1-5.

40. Vassilouthis, J., Ambrose, J.A.E.: Intraventricular meningioma in a child. Surg Neurol 1978; 10: 105-107.

41. Yamashita, J., Handa, H., Iwaki, K., et al.: Recurrence of intracranial meningiomas with special reference to radiotherapy. Surg Neurol 1980; 14:33-40.

42. Zimmerman, H.M.: Brain tumors: Their incidence and classification in man and their experimental production. Ann NY Acad Sci 1969; 159: 337.

Marrero, C.L.; Domínguez J.; Ramos, R.; Gómez, J.; Lourido, J.A.: Meningioma intraventricular. Presentación de un caso en la infancia. Neurocirugía 2005; 16: 523-527.

Correspondencia postal: Jaime Domínguez. Servicio de Neurocirugía. Hospital Universitario Nuestra Señora de Candelaria. Carretera del Rosario S/N. 38010 Santa Cruz de Tenerife. 